ISSN = 1980-993X - doi:10.4136/1980-993X
www.agro.unitau.br/ambi-agua
E-mail: ambi-agua@agro.unitau.br
Tel.: (12) 3625-4116

\title{
Desenvolvimento e avaliação de sonda de TDR para o manejo racional da água em substratos utilizados na produção de mudas florestais (doi:10.4136/ambi-agua.77)
}

\section{Marcelo Leite Conde Elaiuy ${ }^{1}$; Lucas Massayuki Sato ${ }^{1}$; Antonio Claudio Tesla Varallo² Claudinei Fonseca Souza ${ }^{3}$}

\author{
${ }^{1}$ Estudantes de graduação, Departamento de Engenharia Civil - UNITAU \\ E-mail: trangokauk@gmail.com \\ ${ }^{2}$ Professor Mestre, Departamento de Engenharia Civil - UNITAU. E-mail: antonio.varallo@yahoo.com.br \\ ${ }^{3}$ Professor Adjunto, Departamento de Recursos Naturais e Proteção Ambiental - CCA/UFSCar \\ E-mail: cfsouza@cca.ufscar.br
}

\section{RESUMO}

A Reflectometria no Domínio do Tempo (TDR) é uma técnica confiável para determinar a umidade in situ em diferentes tipos de materiais por meio da utilização de sondas. A produção de mudas florestais implica um exaustivo e empírico processo de manejo da água aplicada ao substrato utilizado para o plantio em tubetes. Esse meio de cultivo requer uma análise quanto ao comportamento físico em relação à retenção de água e nutrientes. $\mathrm{O}$ objetivo deste trabalho foi desenvolver e avaliar uma sonda de TDR do tipo coaxial de maneira a permitir o manejo racional da água na produção de mudas florestais. Inicialmente, uma validação física da sonda foi realizada em função dos seguintes parâmetros: coeficiente de reflexão, impedância característica e sensibilidade espacial. Avaliou-se, também, o desempenho da sonda para estimar a umidade volumétrica em condições de laboratório e obteve-se uma curva de calibração para cada tipo de material poroso utilizado. Os resultados demonstraram a viabilidade no uso da sonda de TDR proposta na estimativa da umidade volumétrica no solo e no substrato.

Palavras-chave: TDR; irrigação; manejo da água.

\section{Development and evaluation of TDR probe for water rational management on substrates used in forest seedlings production}

\begin{abstract}
Time Domain Reflectometry (TDR) is a reliable technique to estimate in situ moisture content in different types of materials using probes. The forest seedlings production implies in a comprehensive and empirical process of water management applied to the substrate used for cultivation in dibble-tube. This type of cultivation requires analysis of the physical characteristics of water and nutrients retention of the substrate. The main goal of this research was to develop and evaluate a TDR coaxial probe for rational management of water in the forest seedlings production. Initially, a physical validation of the probe was performed considering the following parameters: reflection coefficient, characteristic impedance and spatial sensitivity. Also, the performance of the probe was evaluated to estimate water content in laboratory conditions and we obtained a calibration curve for each type of porous material used. The results demonstrated the viability of TDR probes to estimate water content in soil and substrates.
\end{abstract}

Keywords: TDR; irrigation; water management. 


\section{INTRODUÇÃO}

Existem no mundo cerca de 3,4 bilhões de hectares de florestas naturais. No início da década, as florestas plantadas totalizavam aproximadamente 130 milhões de hectares, sendo que, aproximadamente, seis milhões correspondiam a plantações de eucalipto. No Brasil, estima-se que as plantações de pinus e eucalipto são de 4,8 milhões de ha, sendo 2.965.880 ha são cultivados com o gênero Eucalyptus (Mora e Garcia, 2000). Pela importância do cenário, que indica expectativas de grandes investimentos, o aumento da produtividade dos maciços florestais torna-se necessário e requer esforços de pesquisas nas mais diversas áreas. Em relação à produção das mudas, a quantificação da necessidade hídrica na sua formação é extremamente importante, pois a falta ou excesso pode limitar o desenvolvimento destas. A falta de água leva ao estresse hídrico (desejável somente na rustificação), além da diminuição na absorção de nutrientes. O excesso pode favorecer a lixiviação dos nutrientes e também proporcionar um micro-clima favorável ao desenvolvimento de doenças, além das questões sócio-ambientais relativas à economia de água e ao acúmulo de lixiviados no solo.

A necessidade do uso racional dos recursos hídricos aliados ao avanço da eletrônica tem contribuído para a intensificação dos estudos do monitoramento da água no solo. $\mathrm{O}$ conhecimento do conteúdo de água no solo é importante na dinâmica de solutos, calor, gases e da própria água no solo. Em escala global, sua avaliação está relacionada com o significante sistema solo- água- planta - atmosfera. A otimização do uso da irrigação baseado na melhoria de informações sobre o conteúdo de água no solo evitará o desperdício desse recurso, observado em diversas situações. Para isso, a determinação in situ do conteúdo de água do solo é de grande interesse. O uso da instrumentação voltada para a obtenção de informações sobre o sistema água-solo é muito importante. Um método de quantificação ideal da água do solo deve basear-se numa propriedade física, dependente apenas do seu conteúdo e produzir uma resposta direta, rápida e confiável.

Diversos são os métodos para a determinação da umidade do solo. O gravimétrico é considerado o método direto padrão.

Os métodos indiretos estimam a umidade a partir de outras propriedades do solo, tais como, termalização de nêutrons, resistência elétrica e técnicas eletromagnéticas. $\mathrm{O}$ método gravimétrico apresenta resultados precisos a um custo relativamente baixo quando usado como técnica laboratorial, mas para medidas no campo, o acondicionamento e transporte das amostras podem reduzir a precisão dos resultados e, ainda, para amostragens repetidas em uma pequena área, podem causar interferência no local em estudo. $\mathrm{O}$ uso da sonda de nêutrons exige um treinamento especial para seu manuseio, principalmente pela presença de fontes radioativas. O bloco de gesso é um instrumento simples e de baixo custo, mas o elemento sensor sofre deterioração com o tempo devido à interação com os compostos físicoquímicos do solo (Selker et al. 1993; Coelho e Or, 1996). Para medidas de umidade do solo em laboratório e em campo, a utilização de técnicas eletromagnéticas possui algumas vantagens quanto ao uso, principalmente pela precisão, possibilidade de multiplicação de leituras, repetição sem destruição da amostra de solo e pela segurança (Topp et al., 1980; Coelho e Or, 1996). Entretanto, a principal desvantagem tem sido o custo ainda alto para os padrões nacionais. No caso dos substratos orgânicos, há uma susceptibilidade à decomposição biológica que modifica suas características físicas e químicas ao longo do tempo, exigindo assim uma análise periódica dessas propriedades. Várias são as proposições para determinação da umidade nos substratos, porém nenhuma consegue satisfazer todas as necessidades e apresentar requisitos básicos para que se torne um método ideal. Dos vários 
métodos existentes, a técnica da TDR vem despertando muito interesse devido à possibilidade de leituras em tempo real de forma automatizada (Souza e Matsura, 2003; Souza et al., 2004).

As técnicas eletromagnéticas baseiam-se no efeito da umidade do solo sobre a velocidade de propagação de pulsos de micro-ondas em cabos condutores envoltos por solo. Esse fenômeno se deve à diferença entre as constantes dielétricas, (Ka [adimensional]), da água, do ar e do material sólido do solo. Na matriz do solo, as constantes dielétricas variam entre 1 e 81 . O ar possui valor mínimo igual a 1 , as partículas sólidas variam entre 3 e 5, e a água possui o valor máximo de 81 (Noborio, 2001). A constante K medida nesse sistema (arsolo-água-atmosfera) é denominada constante dielétrica aparente, assim, conhecendo-se essa variável, pode-se determinar a umidade volumétrica do solo por meio de calibrações obtidas a partir de dados de campo ou de laboratório (Conciani et al., 1996).

Neste trabalho, a correlação entre os parâmetros citados, para a determinação de uma equação de calibração representativa para o solo e os substratos estudados, foi importante no desenvolvimento da sonda coaxial. Para obtenção desses valores há uma equação genérica proposta pelo fabricante do reflectômetro utilizado $\theta$ Topp (Topp et al., 1980). A correlação estatística entre as técnicas gravimétrica e volumétrica também forneceu resultados pertinentes para validação da metodologia utilizada nos ensaios realizados em laboratório.

\section{MATERIAL E MÉTODOS}

A idealização da construção de uma sonda do tipo coaxial proposta neste trabalho foi baseada no conceito de plantio de mudas em tubetes plásticos, amplamente utilizado no mercado, o qual visa à formação de um sistema radicular perfeito, boas condições para transporte, facilidade para o plantio, etc. A sonda coaxial foi projetada para estimar a umidade do solo e do substrato, e suas dimensões são baseadas em uma célula de transmissão coaxial.

O desenvolvimento da sonda levou em conta os critérios observados na literatura: um mínimo de sinal eletromagnético captado, impedância característica inferior a $200 \Omega$ (Souza et al., 2006), uniformidade na distribuição de energia conduzida pela sonda no meio amostrado e mínimo custo (Souza e Matsura, 2002; Hemiovaara, 1993).

\subsection{Sonda coaxial de TDR}

$\mathrm{Na}$ construção da sonda coaxial, foi torneada uma rosca na base de um cilindro de ferro galvanizado com $0,23 \mathrm{~m}$ de extensão e $0,04 \mathrm{~m}$ de diâmetro, onde se rosqueou um "cap" (tampa de PVC) com 1 1 1/4" de diâmetro. Por um orifício desse "cap" passou-se um cabo coaxial RG-58 de $50 \Omega$, cujo condutor interno foi soldado na extremidade inferior de uma haste em aço inox de 0,23 $\mathrm{m}$ de extensão e $0,0045 \mathrm{~m}$ de diâmetro. A malha externa do cabo foi seccionada em duas partes e soldadas em dois pontos paralelos da parede interna do cilindro. Na outra extremidade do cabo foi colocado um conector BNC, ligando a sonda ao reflectômetro. Com o "cap" devidamente rosqueado e a haste já centrada no eixo axial do cilindro, a sonda foi presa num suporte vertical e sua extremidade inferior foi preenchida com resina epóxi até que se atingisse uma altura de $0,05 \mathrm{~m}$ (Offset), fixando sua haste.

O detalhamento da construção da sonda e suas dimensões seguem na Figura 1. 


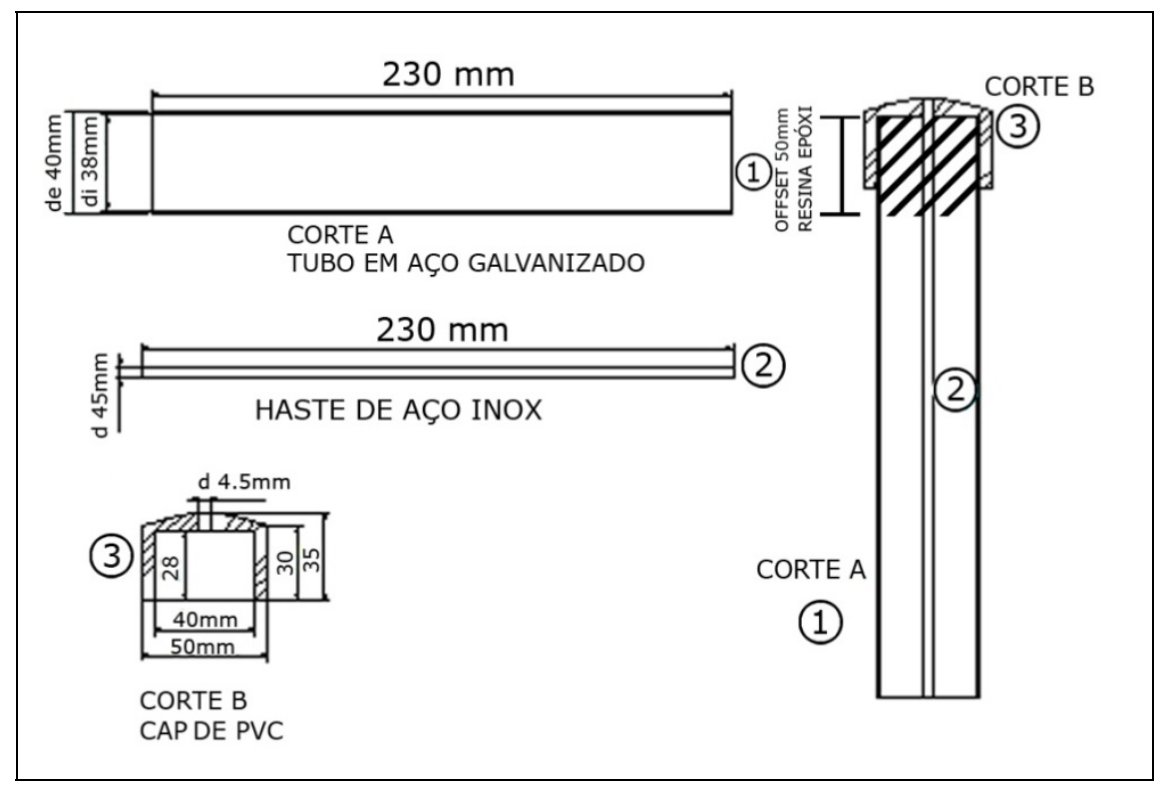

Figura 1. Detalhamento de construção da sonda e suas dimensões.

A sonda coaxial conforme apresentada na Figura 2 foi avaliada fisicamente pelo coeficiente de reflexão, da impedância característica e da sensibilidade espacial.

O coeficiente de reflexão em função da distância percorrida pela onda eletromagnética apontou informações importantes sobre o desempenho das características utilizadas no dimensionamento da sonda, facilitando a interpretação do sinal eletromagnético gerado pelo reflectômetro.

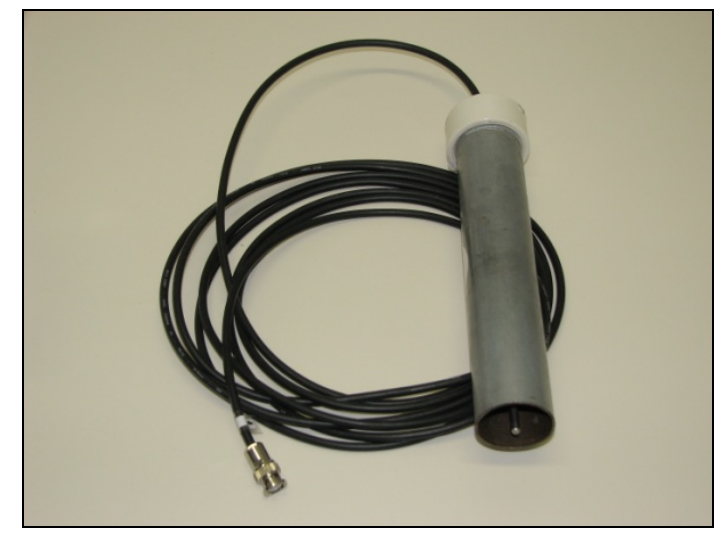

Figura 2. Sonda coaxial de TDR.

A impedância característica $\left(Z_{0}, \Omega\right)$ é o modo de expressar a lei que relaciona a variação de voltagem e corrente com a distância ao longo de uma linha de transmissão uniforme, sendo geralmente utilizada para avaliar o desempenho de sondas de TDR. Em geral, as sondas com $\mathrm{Z}_{0}$ superior a $200 \Omega$ são limitantes para a determinação da umidade do solo, pois, a impedância característica pode causar um aumento na atenuação do sinal da onda eletromagnética a ser analisado durante a distância percorrida, prejudicando a interpretação desse sinal e dificultando que a resina epóxi empregada na confecção do cabeçote da sonda seja utilizada como o marcador do início desta. Entretanto, alguns autores descrevem bons resultados utilizando sondas com valores de $Z_{0}$ superiores ao recomendado. Nesses casos, é comum o uso de componentes eletrônicos para facilitar a interpretação do sinal eletromagnético. 
Segundo Zegelin et al. (1989) e Heimovaara (1993), pode-se medir $Z_{0}$ por determinação do coeficiente de reflexão da sonda (p), isso quando ela está envolta por um material dielétrico uniforme.

$$
Z_{0}=Z_{u} \cdot K^{0,5} \cdot \frac{(1+p)}{(1-p)}
$$

sendo,

$$
p=\left(\frac{V_{1}}{V_{0}}\right)-1
$$

$\mathrm{V}_{0}=$ Amplitude do sinal eletromagnético gerado pela TDR, (m);

$\mathrm{V}_{1}=$ Amplitude do sinal eletromagnético depois da reflexão do início da sonda, (m);

$\mathrm{Zu}=$ Impedância no cabo $\sim 50 \Omega$;

$\mathrm{K}=$ Constante dielétrica, obtida em água destilada (Souza et. al., 2004).

Para a determinação dos parâmetros mencionados, utilizou-se um Reflectômetro (TDR 100 - Campbell Scientific, Figura 3) equipado com uma interface RS 232, em que foi analisado o sinal eletromagnético automaticamente pelo programa WinTDR 6.0 (Utah State University, Logan-Utah).

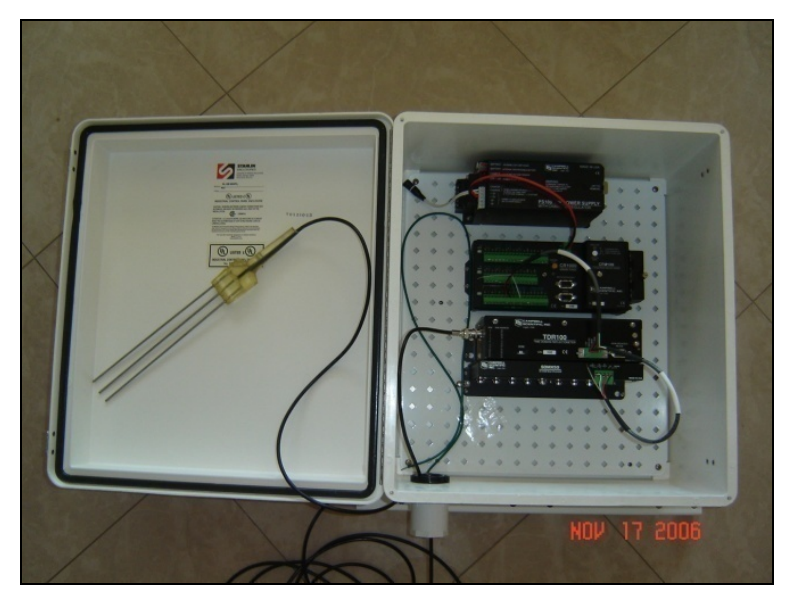

Figura 3. Reflectômetro TDR 100.

A sensibilidade espacial é um conceito introduzido por Knight (1992), o qual descreve a uniformidade de distribuição de energia emitida pela onda eletromagnética em função da água distribuída entre os condutores interno e externo da sonda de TDR. Assim, quanto maior a uniformidade de distribuição melhor será a representatividade da umidade volumétrica espacialmente medida. Knight (1992) sugere uma relação prática para auxiliar na avaliação de sondas de TDR:

$$
\frac{a}{b} \geq 0,1
$$

sendo,

$\mathrm{a}=$ diâmetro do condutor interno;

$\mathrm{b}=$ diâmetro do condutor externo. 


\subsection{Caracterização do solo e do substrato utilizado}

O solo utilizado para avaliação da sonda coaxial pertence ao grupo Latossolo VermelhoAmarelo, coletado no campo do Departamento das Ciências Agrárias da Universidade de Taubaté-SP, da camada de 0 - 0,20 m, com uma textura média do tipo areno-argiloso com as características físico-hídricas apresentadas na Tabela 1.

Tabela 1. Características físico-hídricas do solo.

\begin{tabular}{|c|c|c|c|c|c|c|c|c|}
\hline $\mathrm{CC}$ & PMP & $\mathbf{P}$ & DS & DP & VIB & Areia & Silte & Argila \\
\hline & $\left.\mathrm{m}^{3} / \mathrm{m}^{3}\right)$ & & \multicolumn{2}{|c|}{$\left(\mathrm{g} / \mathrm{cm}^{3}\right)$} & $(\mathrm{mm} / \mathrm{h})$ & & $(\%)$ & \\
\hline 0,34 & 0,22 & 0,56 & 1,4 & 2,7 & 7,2 & 59 & 13 & 28 \\
\hline
\end{tabular}

Os substratos submetidos aos estudos foram:

a) Plantmax ${ }^{\circledR}$ : substrato comercial tendo como principais componentes a casca de pinus carbonizada e vermiculita, sendo bastante difundido entre os produtores de mudas de hortaliças.

b) Fibra de coco: substrato comercial Golden Mix tipo 80, sem aditivação, granulado da empresa Amafifra, localizada em Anamindeua - PA. Substrato elaborado a partir do mesocarpo do coco, possuindo textura fina, indicado para formação de mudas em bandejas e tubetes.

Foi utilizado um arranjo para a determinação da densidade úmida dos substratos (Figura 4) empregando-se o método descrito por (Hoffman, 1970), utilizado pela Federação dos Institutos para a Pesquisa e Análises Agrícolas da Alemanha (VDLUFA) para análise de substratos hortícolas (Röber e Schaller, 1985). Nessa metodologia, seis amostras de cada substrato no estado fresco são colocadas em proveta de plástico transparente e graduada de $500 \mathrm{ml}$ de capacidade, a qual deixa-se cair por 10 vezes consecutivas sob ação de seu próprio peso de uma altura de $10 \mathrm{~cm}$. Com ajuda de uma espátula nivelou-se o volume obtido (ml). Em seguida, pesou-se o material fresco (g) e colocou-se em estufa a $65^{\circ} \mathrm{C}$, por 48 horas ou até peso constante. Os valores das densidades de volume (média de três amostras) foram obtidos aplicando-se a seguinte fórmula:

DensidadeÚmida $\left(K g . m^{-3}\right)=\frac{\operatorname{MassaÚmida}(\mathrm{g})}{\operatorname{Volume}\left(\mathrm{cm}^{3}\right)} \times 1000$

\subsection{Determinações da constante dielétrica aparente e da umidade volumétrica}

Para efetuar as determinações da constante dielétrica aparente $(\mathrm{Ka})$ e da umidade volumétrica ( $\theta$ Topp) com a sonda coaxial, seguiu-se o procedimento descrito por Tommaselli e Bacchi (2001). Nessa metodologia, as etapas de umedecimento com adição de $50 \mathrm{~mL}$ de água por etapa foram continuamente repetidas até atingir as umidades próximas à da saturação conforme a seguinte descrição: metade da amostra de solo coletada, cerca de $2 \mathrm{~L}$, foi esparramada em uma mesa formando uma fina camada. Através de um pulverizador adicionou-se cerca de metade da água desejada $(25 \mathrm{~mL})$. A seguir, a outra metade de solo foi esparramada sobre a metade anterior pulverizando-se com água até completar os $50 \mathrm{~mL}$. 


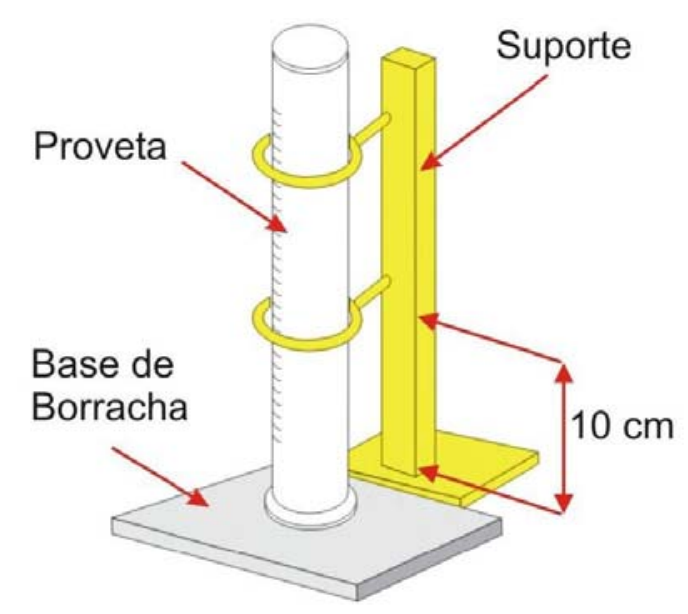

Figura 4. Arranjo para determinação da densidade dos substratos.

O solo foi bem misturado e peneirado de três a quatro vezes, em peneira de malha de $2 \mathrm{~mm}$, obtendo-se sua homogeneização. Após o umedecimento e homogeneização, o solo foi colocado em uma sonda coaxial com cerca de 0,225 L de volume, compactando-se de modo a manter a sua densidade o mais uniforme possível. A sonda foi conectada ao reflectômetro e foram lidos os parâmetros da umidade volumétrica ( $\theta$ Topp) e da constante dielétrica aparente (Ka) para cada umedecimento. Em seguida, uma amostra deste mesmo solo foi colocada em uma cápsula e pesada para depois ser mantida em estufa à $105^{\circ} \mathrm{C}$ por 48 horas, para obtenção da umidade gravimétrica. Para cada determinação de umidade volumétrica foi obtido um parâmetro de umidade gravimétrica. Os substratos foram submetidos aos mesmos procedimentos para obtenção das umidades e da constante dielétrica aparente.

As estimativas determinadas pela sonda proposta foram confrontadas com o método padrão (Gravimetria), utilizando-se um diagrama de dispersão e um coeficiente de correlação.

\section{RESULTADOS E DISCUSSÃO}

\subsection{Coeficiente de reflexão}

A Figura 5 descreve o coeficiente de reflexão em função da distância percorrida para a sonda proposta. Observa-se a interpretação da onda eletromagnética sendo, $\mathrm{X}_{1}$ a distância no início da sonda, $\mathrm{X}_{2}$ a distância no final da sonda, $\mathrm{V}_{0}$ a amplitude do sinal eletromagnético gerado pela TDR e $\mathrm{V}_{1}$ a amplitude do sinal depois da reflexão do início da sonda. Por meio desses parâmetros, pode-se determinar a impedância (Equação 1) e a constante dielétrica pela equação abaixo (Hook e Livingston, 1995). A constante dielétrica é dada por:

$$
K a=\left(\frac{\Delta x}{V p . L}\right)^{2}
$$

sendo,

$\Delta \mathrm{x}=$ Distância percorrida pela onda eletromagnética, (m);

$\mathrm{Vp}=$ Velocidade de propagação, 0,99 (99\% de c);

$\mathrm{c}=$ Velocidade da luz, $3 \times 10^{8} \mathrm{~m} . \mathrm{s}^{-1}$;

$\mathrm{L}=$ Comprimento da haste, $(\mathrm{m})$. 


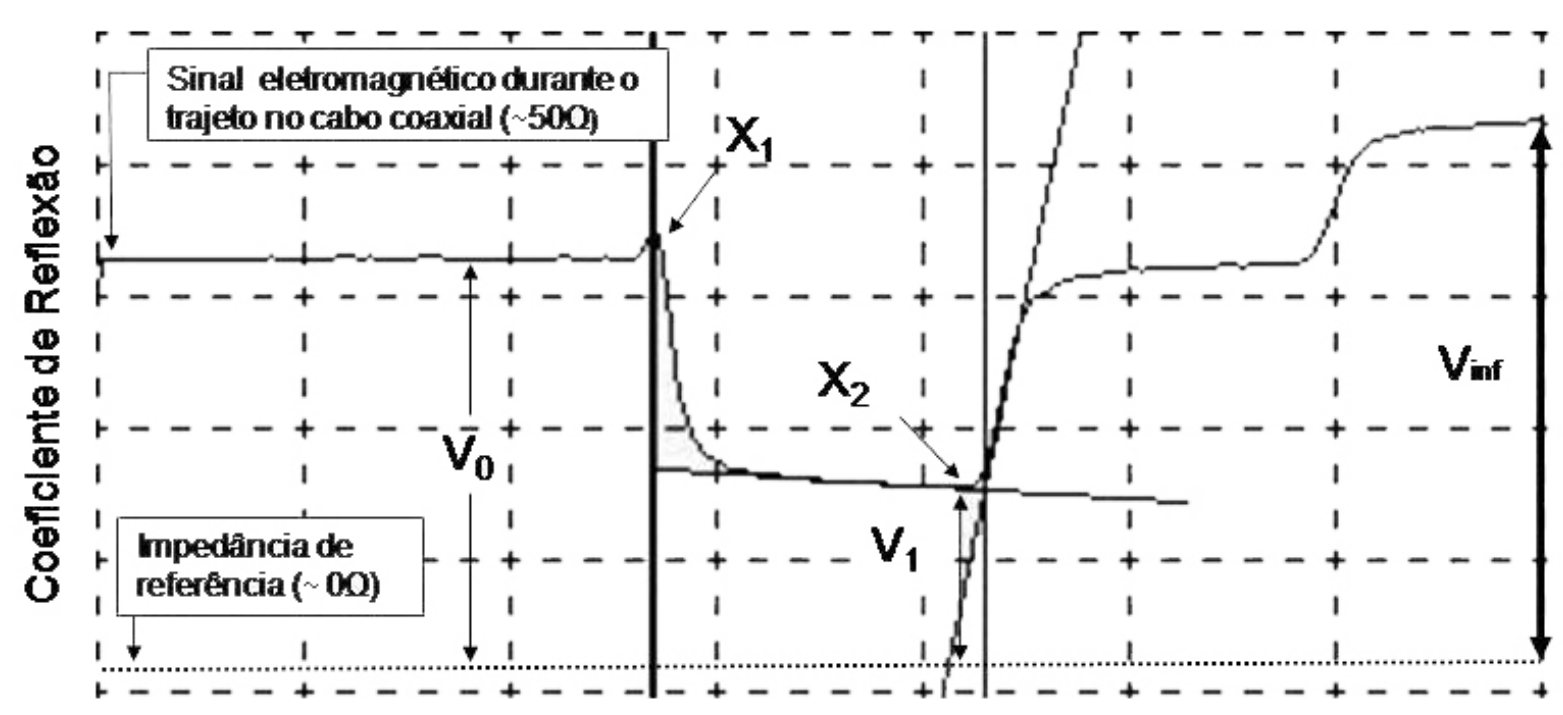

\section{Distância (m)}

Figura 5. Coeficiente de reflexão em função da distância do sinal eletromagnético percorrido na sonda.

\subsection{Impedância característica e sensibilidade espacial}

A Tabela 2 analisa as dimensões geométricas utilizadas e apresenta os valores de impedância tanto medida como calculada inferior ao recomendado $200 \Omega$ (Zegelin et al., 1989) e com sensibilidade espacial $\geq 0,1$ (Knight, 1992). Os resultados observados demonstram que a sonda proposta possui características dimensionais apropriadas para atender às atribuições físicas desejadas em uma sonda de TDR, quando comparada com outros modelos de sondas (Souza et al., 2006).

Tabela 2. Dimensões geométricas, impedância medida e calculada para diferentes configurações de sondas de TDR.

\begin{tabular}{|c|c|c|c|c|c|c|}
\hline & $\begin{array}{r}\text { Diâm } \\
(m)\end{array}$ & & $\begin{array}{c}\text { Sensibilidade } \\
\text { Espacial }\end{array}$ & \multirow{2}{*}{$\begin{array}{c}\text { Volume } \\
\left(\mathrm{m}^{3}\right)\end{array}$} & \multirow{2}{*}{$\begin{array}{c}\text { Impedância } \\
\text { Calculada } \\
\text { Zo }(\Omega)\end{array}$} & \multirow{2}{*}{$\begin{array}{c}\text { Impedância } \\
\text { Medida } \\
Z o(\Omega)\end{array}$} \\
\hline & \multicolumn{2}{|c|}{ Interno, Externo, } & $a / b$ & & & \\
\hline Sonda coaxial & 0,0045 & 0,04 & 0,11 & 0,0002 & 131,08 & 172,67 \\
\hline Sonda contínua & 0,003 & 0,027 & 0,11 & 0,0001 & 131,83 & 197,00 \\
\hline Sonda muti-haste & 0,006 & 0,058 & 0,11 & 0,0005 & 132,43 & 168,00 \\
\hline
\end{tabular}

\subsection{Cálculo do erro padrão da estimativa}

A Tabela 3 mostra a avaliação estatística do erro padrão da estimativa na determinação da umidade (Spiegel, 1985):

$\mathrm{S}= \pm \sqrt{\frac{\sum\left(Y-Y_{e s t}\right)^{2}}{n}}$

em que, Y é o valor medido e $\mathrm{Y}_{\text {est }}$ o estimado pela equação utilizada (Topp), e $n$ o número de amostras utilizadas. Os dados mostram que o erro padrão é relativamente pequeno para todos 
os materiais analisados, não interferindo dessa forma nos resultados de determinação da umidade, podendo ser facilmente ajustados com uma calibração específica.

Tabela 3. Erro padrão da estimativa na determinação da umidade.

\begin{tabular}{|c|c|}
\hline Amostra & S: Erro padrão da estimativa $\left(\mathrm{m}^{3} \mathrm{~m}^{-3}\right)$ \\
\hline Solo & $\pm 0,039$ \\
\hline Plantmax ${ }^{\circledR}$ & $\pm 0,109$ \\
\hline Fibra de coco & $\pm 0,087$ \\
\hline
\end{tabular}

\subsection{Determinação da densidade úmida}

Os valores das densidades úmidas obtidos em laboratório pelo método descrito por Hoffman (1970) estão apresentados na Tabela 4. Esses valores foram utilizados na determinação da umidade gravimétrica dos substratos. No caso do solo, para a obtenção desse mesmo parâmetro, a densidade utilizada está apresentada na Tabela 1.

Tabela 4. Valores das densidades úmidas obtidos em laboratório.

\begin{tabular}{lc}
\hline Amostra & Densidade úmida $\left(\mathbf{K g} \cdot \mathbf{m}^{-3}\right)$ \\
\hline Plantmax ${ }^{\circledR}$ & 586 \\
Fibra de coco & 131 \\
\hline
\end{tabular}

\subsection{Estimativa da umidade e da constante dielétrica em condições de laboratório}

Com os resultados numéricos das umidades apresentados nas Tabelas de 5 a 7 , foi feita a correlação entre os valores obtidos pelas duas técnicas para cada material, a fim de comparar o método gravimétrico com a técnica da TDR. A partir dos resultados de Ka e $\theta$, pôde-se determinar as equações representativas para as condições em estudo.

Tabela 5. Resultados do Latossolo vermelho-amarelo obtidos em condições de laboratório.

\begin{tabular}{cccc}
\hline Leitura & $\begin{array}{c}\text { Constante } \\
\text { Dielétrica, } \boldsymbol{K a}\end{array}$ & \multicolumn{2}{c}{ Umidade $\left(\mathbf{m}^{\mathbf{3}} \cdot \mathbf{m}^{-\mathbf{3}}\right)$} \\
\cline { 2 - 4 } & 4,393 & $\mathbf{\theta T o p p}$ & Gravimétrica \\
\hline $1^{\mathrm{a}}$ & 5,061 & 0,067 & 0,023 \\
$2^{\mathrm{a}}$ & 5,602 & 0,081 & 0,032 \\
$3^{\mathrm{a}}$ & 6,085 & 0,093 & 0,079 \\
$4^{\mathrm{a}}$ & 6,446 & 0,103 & 0,078 \\
$5^{\mathrm{a}}$ & 8,030 & 0,115 & 0,091 \\
$6^{\mathrm{a}}$ & 9,182 & 0,148 & 0,119 \\
$7^{\mathrm{a}}$ & 11,388 & 0,176 & 0,138 \\
$8^{\mathrm{a}}$ & 12,833 & 0,214 & 0,154 \\
$9^{\mathrm{a}}$ & 14,900 & 0,241 & 0,186 \\
$10^{\mathrm{a}}$ & 18,201 & 0,277 & 0,226 \\
$11^{\mathrm{a}}$ & & 0,323 & 0,306 \\
\hline
\end{tabular}

\subsection{Correlação e equação de calibração}

As Figuras de 6 a 8 mostram a correlação entre os valores da umidade volumétrica ( $\theta$ Topp) e os da umidade gravimétrica. Observa-se que o desempenho entre as técnicas é praticamente o mesmo. Há um desalinhamento entre os pontos correlacionados dessas figuras, o que demonstra que a água foi retida pelas partículas do solo/substrato e não permanece ou não se encontra mais livre nesse meio. Isso implica uma discrepância entre os valores da umidade obtidos pela técnica da TDR e os valores obtidos pelo método gravimétrico. De acordo com Valero (2006), a afirmativa de TOPP et al. (1980) de que a constante dielétrica aparente dos meios porosos depende apenas do seu conteúdo de água, seria de se esperar que o seu modelo tivesse o mesmo tipo de comportamento em cada um dos 
substratos estudados. Contudo, observa-se que o comportamento do modelo é distinto para cada um dos substratos, demonstrando que a constante dielétrica (Ka) dos substratos depende de suas propriedades intrínsecas.

Tabela 6. Resultados do substrato Plantmax ${ }^{\circledR}$ obtidos em condições de laboratório.

\begin{tabular}{cccc}
\hline Leitura & $\begin{array}{c}\text { Constante } \\
\text { Dielétrica, Ka }\end{array}$ & \multicolumn{2}{c}{ Umidade $\left.\mathbf{~ ( ~}^{\mathbf{3}} \cdot \mathbf{m}^{-3}\right)$} \\
\cline { 2 - 4 } & 5,597 & 0,096 & Gravimétrica \\
\hline $1^{\text {a }}$ & 6,345 & 0,112 & 0,059 \\
$2^{\text {a }}$ & 7,178 & 0,130 & 0,084 \\
$3^{\text {a }}$ & 8,372 & 0,156 & 0,124 \\
$4^{\mathrm{a}}$ & 9,760 & 0,182 & 0,128 \\
$5^{\text {a }}$ & 14,900 & 0,271 & 0,163 \\
$6^{\mathrm{a}}$ & 16,800 & 0,307 & 0,177 \\
$7^{\mathrm{a}}$ & 20,144 & 0,340 & 0,194 \\
$8^{\mathrm{a}}$ & 21,458 & 0,368 & 0,219 \\
$9^{\mathrm{a}}$ & 27,507 & 0,457 & 0,240 \\
$10^{\mathrm{a}}$ & 32,319 & 0,481 & 0,259 \\
$11^{\mathrm{a}}$ & & & 0,287 \\
\hline
\end{tabular}

Tabela 7. Resultados do substrato Fibra de coco obtidos em condições de laboratório.

\begin{tabular}{|c|c|c|c|}
\hline \multirow[t]{2}{*}{ Leitura } & \multirow{2}{*}{$\begin{array}{c}\text { Constante } \\
\text { Dielétrica, Ka }\end{array}$} & \multicolumn{2}{|c|}{ Umidade $\left(\mathrm{m}^{3} \cdot \mathrm{m}^{-3}\right)$} \\
\hline & & өТорр & Gravimétrica \\
\hline $1^{\mathrm{a}}$ & 2,538 & 0,017 & 0,021 \\
\hline $2^{\mathrm{a}}$ & 3,216 & 0,038 & 0,042 \\
\hline $3^{\mathrm{a}}$ & 4,287 & 0,063 & 0,062 \\
\hline $4^{\mathrm{a}}$ & 4,638 & 0,073 & 0,066 \\
\hline $5^{\mathrm{a}}$ & 5,992 & 0,104 & 0,088 \\
\hline $6^{\mathrm{a}}$ & 6,786 & 0,123 & 0,097 \\
\hline $7^{\mathrm{a}}$ & 7,936 & 0,149 & 0,111 \\
\hline $8^{\mathrm{a}}$ & 9,764 & 0,186 & 0,140 \\
\hline $9^{a}$ & 10,946 & 0,209 & 0,170 \\
\hline $10^{\mathrm{a}}$ & 11,425 & 0,220 & 0,173 \\
\hline $11^{\mathrm{a}}$ & 14,652 & 0,270 & 0,201 \\
\hline $12^{\mathrm{a}}$ & 16,702 & 0,302 & 0,228 \\
\hline $13^{\mathrm{a}}$ & 19,099 & 0,332 & 0,235 \\
\hline $14^{\mathrm{a}}$ & 23,035 & 0,382 & 0,268 \\
\hline $15^{\mathrm{a}}$ & 25,216 & 0,401 & 0,274 \\
\hline $16^{\mathrm{a}}$ & 29,298 & 0,439 & 0,294 \\
\hline $17^{\mathrm{a}}$ & 32,952 & 0,466 & 0,331 \\
\hline $18^{\mathrm{a}}$ & 39,090 & 0,505 & 0,342 \\
\hline $19^{\mathrm{a}}$ & 41,215 & 0,515 & 0,363 \\
\hline
\end{tabular}

Os resultados deste trabalho dão evidências de que, além da água, a distribuição e o tamanho de partículas e a superfície específica são características que podem influenciar no valor da constante dielétrica do material, como no caso dos substratos orgânicos em estudo. 


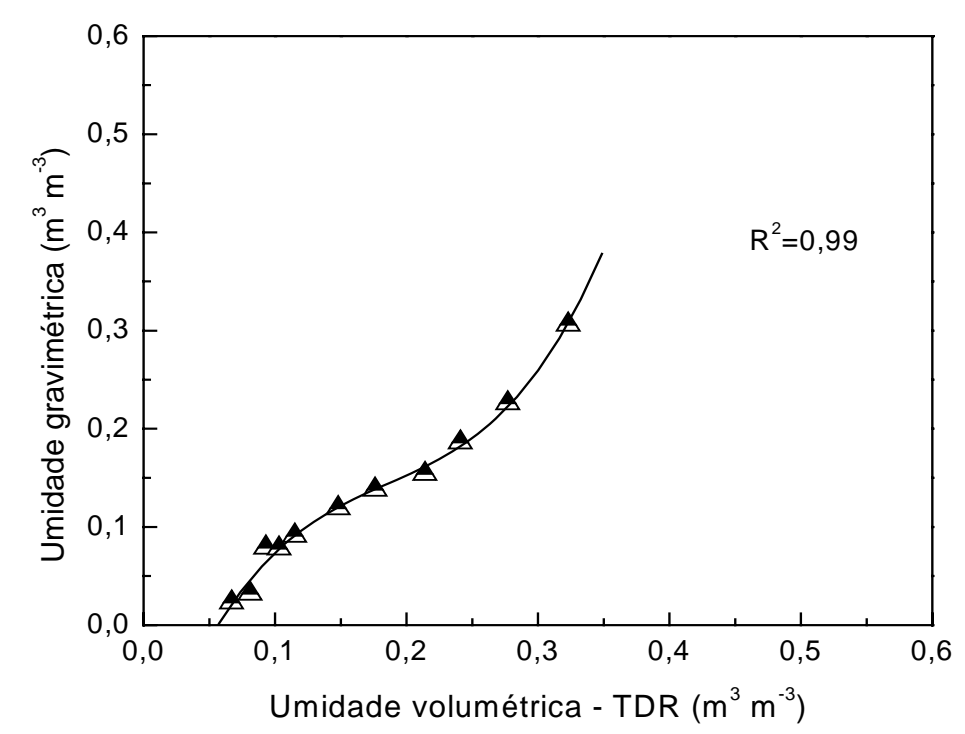

Figura 6. Correlação entre a técnica da TDR e a gravimétrica para o solo.

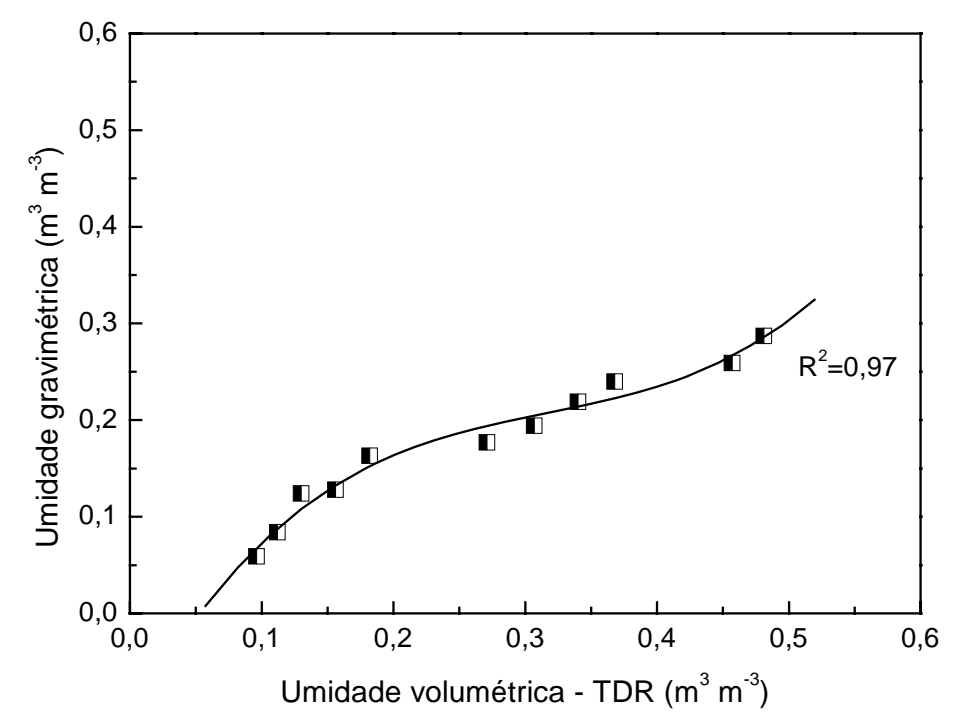

Figura 7. Correlação entre a técnica da TDR e a gravimétrica para o substrato plantmax ${ }^{\circledR}$.

Portanto, de acordo com os resultados os valores da constante dielétrica aparente (Ka) e da umidade gravimétrica do solo e dos substratos $\left(\mathrm{m}^{3} \cdot \mathrm{m}^{-3}\right)$ puderam ser ajustados por meio das equações polinomiais cúbicas obtidas na calibração da TDR, apresentadas na Tabela 8.

Tabela 8. Equações polinomiais cúbicas obtidas na calibração da TDR.

\begin{tabular}{llc}
\hline Material & \multicolumn{1}{c}{ Equações } & $\mathbf{R}^{\mathbf{2}}$ \\
\hline Solo & $\theta=-1,83931 E-6 K a^{3}-3,5281 E-4 K a^{2}+0.0274 K a-0,4802$ & 0,99 \\
Plantmax ${ }^{\circledR}$ & $\theta=-3,37048 E-6 K a^{3}-4,93629 E-5 K a^{2}+0,2058 K a-0,01406$ & 0,99 \\
Fibra de Coco & $\theta=4,5707 E-K a^{3}-5,68676 E-4 K a^{2}+0,02947 K a-0,05237$ & 0,99 \\
\hline
\end{tabular}




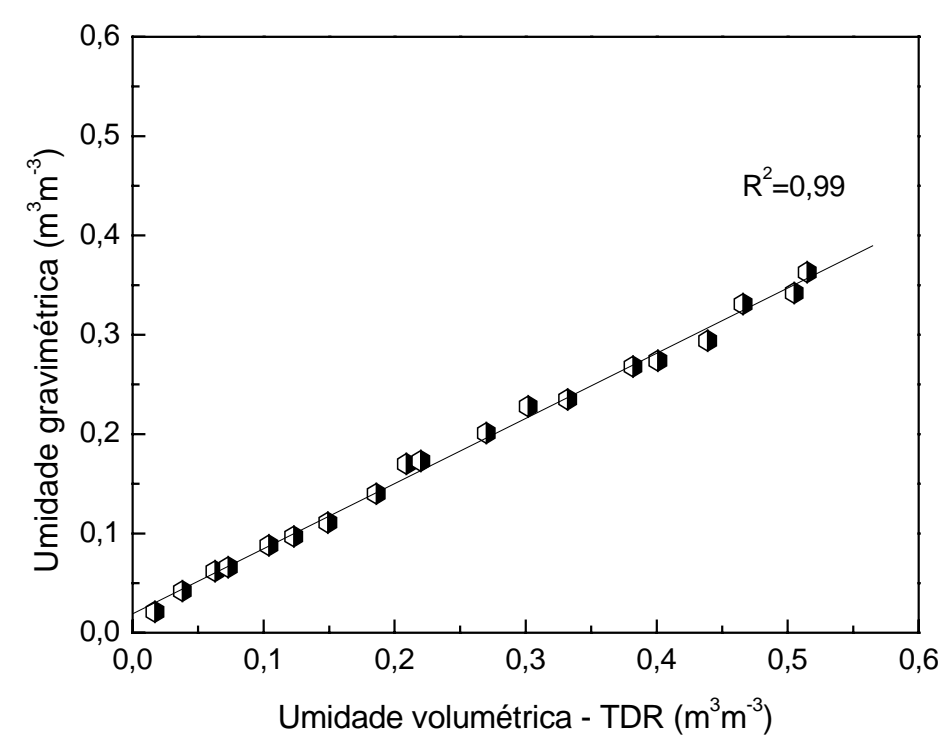

Figura 8. Correlação entre a técnica da TDR e a gravimétrica para o substrato de fibra de coco.

\subsection{Estimativa da regressão linear}

Verificou-se que os coeficientes de correlação entre os métodos de determinação da umidade no solo e nos substratos são satisfatórios e, que apenas o coeficiente angular da equação de regressão do solo ficou próximo a 1, conforme os dados apresentados nas Figuras de 9 a 11. Entretanto, os resultados comprovam existir uma associação linear entre as técnicas e indicam uma variação direta no valor da umidade volumétrica para um valor de umidade gravimétrica. Assim, com uma calibração, a técnica da TDR poderá ter um desempenho semelhante ao método gravimétrico para obtenção dos valores da umidade presente nos materiais analisados. Por outro lado, essa técnica promove agilidade na estimativa, uma vez que, o tempo envolvido na obtenção da umidade é extremamente inferior a essa determinação realizada pelo método padrão, proporcionando contribuições rápidas e precisas, de forma que as informações possam ser utilizadas para auxiliar nas melhores decisões possíveis dentro das condições de incerteza.

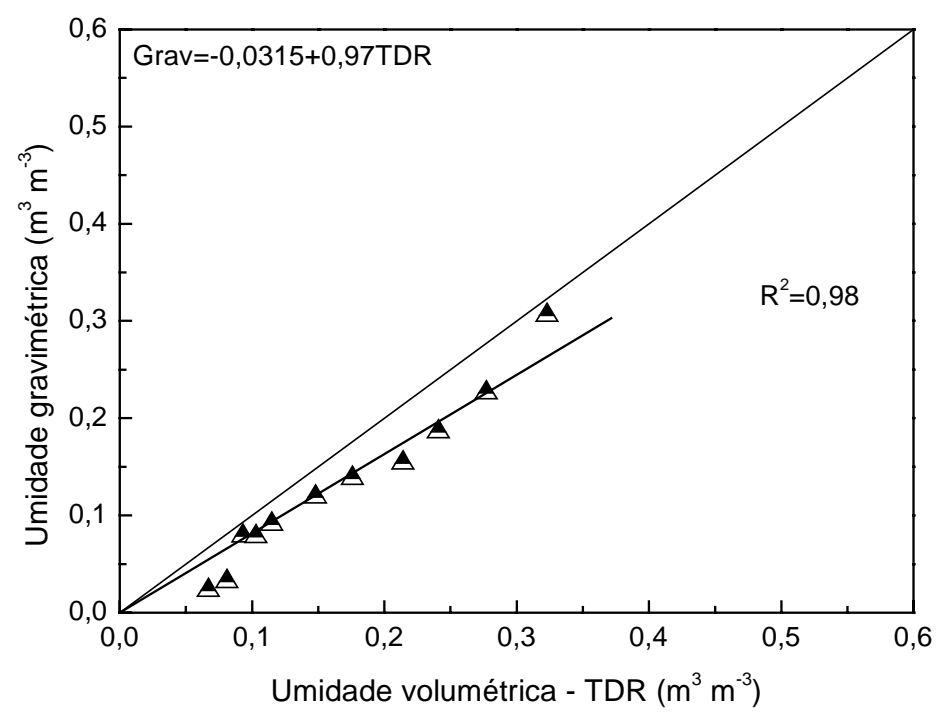

Figura 9. Regressão linear entre a umidade gravimétrica e volumétrica do solo. 


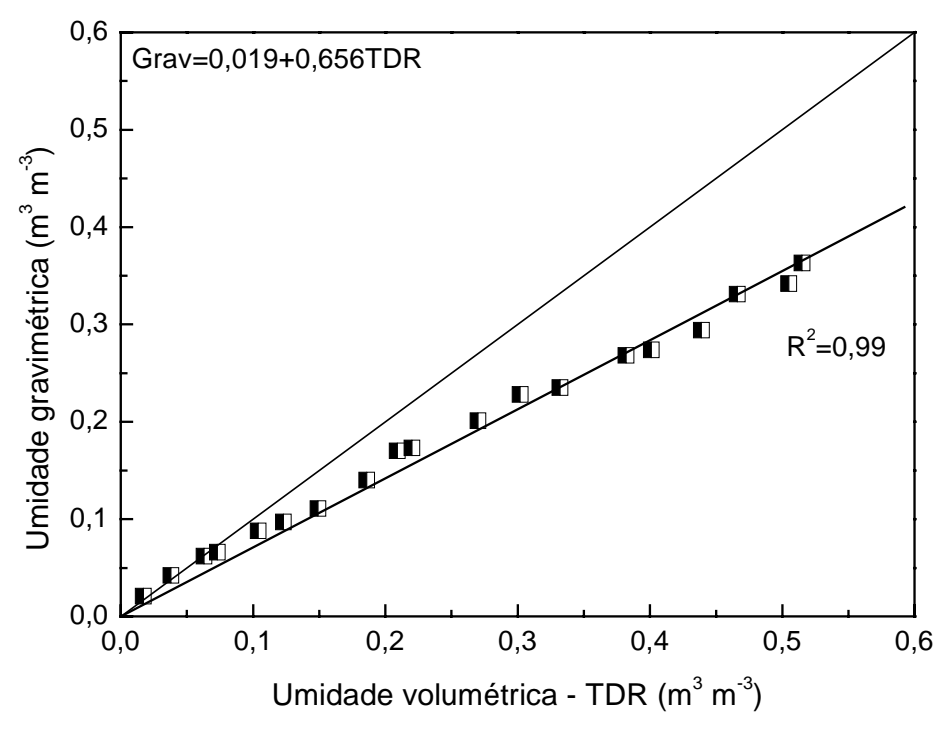

Figura 10. Regressão linear entre a umidade gravimétrica e volumétrica do substrato plantmax ${ }^{\circledR}$.

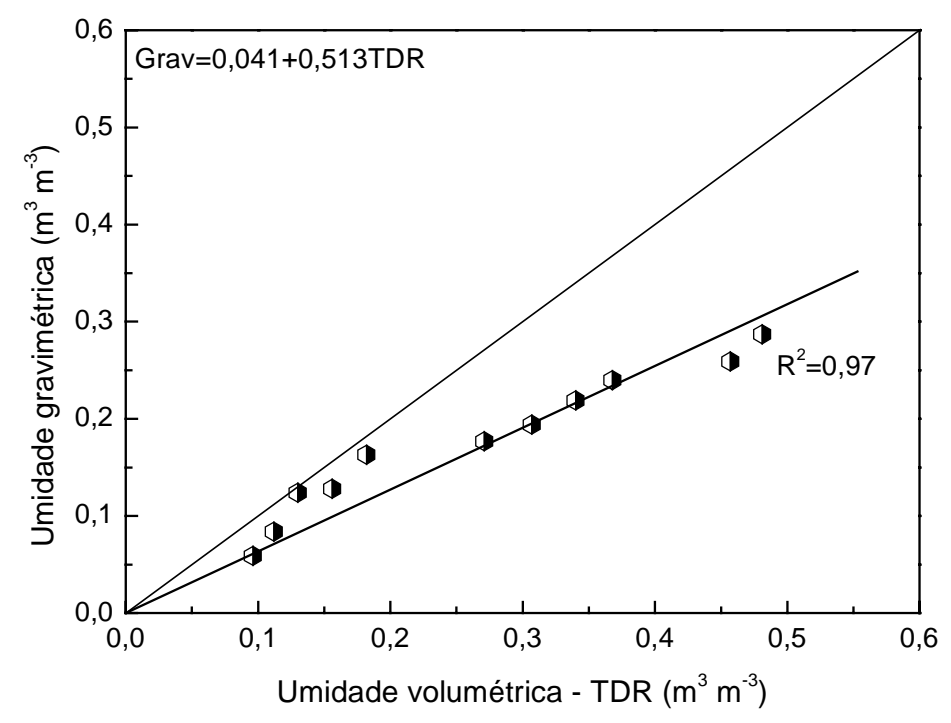

Figura 11. Regressão linear entre a umidade gravimétrica e volumétrica do substrato fibra de coco.

\section{CONCLUSÃO}

Diante dos resultados obtidos, pôde-se inferir que a sonda proposta apresenta o conjunto de critérios físicos que a qualificam como uma alternativa viável na obtenção da umidade do solo e dos substratos analisados, por meio da técnica indireta da reflectometria no domínio do tempo. As curvas de calibração da $\mathrm{Ka}$ e de umidade volumétrica obtidas em cada substrato mostraram-se adequadas e estatisticamente significativas no intervalo de aplicabilidade, ou seja, na faixa de água facilmente disponível nos substratos estudados.

A equação de calibração proposta por Topp et al. (1980) que acompanha equipamento (TDR 100) não é aplicável para estimativa de umidade volumétrica nos substratos estudados, necessitando de uma calibração individual para cada um deles. 


\section{AGRADECIMENTOS}

Os autores agradecem à Fundação de Amparo à Pesquisa do Estado de São Paulo pelos auxílios concedidos. (Proc. Número 05/00906-3 e 06/03241-5).

\section{REFERÊNCIAS}

COELHO, E. F.; OR, D. Flow and uptake patterns affecting soil water sensor placement for drip irrigation management. Transactions of the ASAE, St. Joseph, v. 39, p. 2007 2016, 1996.

CONCIANI, W.; HERRMANN, P. S. P.; MACHADO, S. L.; SOARES, M. M. O uso da técnica de reflectometria no domínio do tempo (TDR) para determinação da umidade do solo in situ. Revista Solos e Rochas, São Paulo, v. 19, p. 189-199, 1996.

HEIMOVAARA, T. J. Design of triple-wire Time Domain Reflectometry probes in practice and theory. Soil Science Society of America Journal, Madison, v. 57, p. 1410 - 1417, 1993.

HOFFMAN, G. Verbindliche Methoden zur Untersuchung von TKS und Gartnerischen Erden. Mitteilubngen der VDLFA, Heft, v. 6, p. 129-153, 1970.

HOOK, W. R; LIVINGSTON, N. J. Propagation velocity errors in time domain reflectometry measurements of soil water. Soil Science Society of America Journal, Madison, v. 28, p. $2345-2352,1995$.

KNIGHT, J. H. Sensitivity of time domain reflectometry measurements to lateral variations in soil water content. Water Resources Research, Washington, v. 28, p. 2345 - 2352, 1992.

MORA, A. L.; GARCIA, C. H. A cultura do eucalipto no Brasil. São Paulo: Verso e Reverso Comunicações, 2000. 112 p.

NOBORIO, K. Measurement of soil water content and electrical conductivity by time domain reflectometry: a review. Computers and Electronics in Agriculture, Amsterdam, v. 31, p. 213-237, 2001.

RÖBER, R.; SCHALLER, K. Pflazenermahrung im Gartenbau. Stuttgart: Ulmer, 1985. 350 p.

SELKER, J. R.; GRAFF, L.; STEENHUIS, T. Noninvasive time domainreflectometry moisture measurement probe. Soil Science Society of America Journal, Madison, v. 57, p. 934-936, 1993.

SOUZA, C. F.; MATSURA, E. E. Avaliação de sondas multi-haste segmentadas para o monitoramento da umidade do solo por meio da técnica de TDR. Revista Brasileira de Engenharia Agrícola e Ambiental, Campina Grande, v. 6, p. 63-68, 2002.

SOUZA, C. F.; MATSURA, E. E. Determination of the wetting front in drip irrigation using TDR Multi-wire probe. Agricultural Water Management, Amsterdam, v. 59, p. 205216, 2003.

SOUZA, C. F.; OR, D.; MATSURA, E. E. A variable-volume TDR probe for measuring water content in large soil volumes. Soil Science Society of America Journal, Madison, v. 68, p. 25-31, 2004. 
SOUZA, C. F.; FOLEGATTI, M. V.; MATSURA, E. E.; OR, D.; COELHO, E. F. Sondas de TDR para a estimativa da umidade e da condutividade elétrica do solo. Revista Irriga, Botucatu, v. 11, p. 12-25, 2006.

SPIEGEL, M. R. Estatística. 2. ed. Rio de Janeiro: Mc Graw-Hill, 1985. 454p.

TOMMASELLI, J. T. C.; BACCHI, O. O. S. Calibração de um equipamento de TDR para medida de umidade de solos. Pesquisa Agropecuária Brasileira, Brasília, v. 36, p. 1145-1154, 2001.

TOPP, G. C.; DAVIS, J. L.; ANNAN, A. P. Electromagnetic determination of soil water content: measurements in coaxial transmission lines. Water Resources Research, Washington, v. 16, p. 574-582, 1980.

VALERO, R. M. M. Uso da técnica da "TDR" na estimativa da umidade e condutividade elétrica em substratos orgânicos. 89f. 2006. Dissertação (Mestrado em Engenharia Agrícola) - Faculdade de Engenharia Agrícola, Universidade Estadual de Campinas, Campinas, 2006.

ZEGELIN, S. J.; WHITE, I.; JENKINS, D. R. Improved fields probes for soil water content and electrical conductivity measurements using TDR. Water Resources Research, Washington, v. 25, p. 2367-2376, 1989. 